\title{
Radiometric and noise characteristics of InAs-rich T2SL MWIR pin photodiodes
}

\author{
E. Giard \\ edouard.giard@onera.fr \\ R. Taalat \\ rachid.taalat@ies.univ-montp2.fr
}

\section{Delmas}

marie.delmas@ies.univ-montp2.fr

J.-B. Rodriguez

rodriguez@ies.univ-montp2.fr

\section{P. Christol}

philippe.christol@ies.univ-montp2.fr

\section{Ribet-Mohamed \\ isabelle.ribet@onera.fr}

\author{
ONERA/DOTA, Chemin de la Hunière, 91761 Palaiseau Cedex, France \\ Institut d'Electronique du Sud, UMR-CNRS 5214, Université Montpellier 2, Place Eugne Bataillon, \\ 34095 Montpellier Cedex 5, France \\ Institut d'Electronique du Sud, UMR-CNRS 5214, Université Montpellier 2, Place Eugne Bataillon, \\ 34095 Montpellier Cedex 5, France \\ Institut d'Electronique du Sud, UMR-CNRS 5214, Université Montpellier 2, Place Eugne Bataillon, \\ 34095 Montpellier Cedex 5, France \\ Institut d'Electronique du Sud, UMR-CNRS 5214, Université Montpellier 2, Place Eugne Bataillon, \\ 34095 Montpellier Cedex 5, France \\ ONERA/DOTA, Chemin de la Hunière, 91761 Palaiseau Cedex, France
}

We present a full characterization of the radiometric performances of a type-II InAs/GaSb superlattice pin photodiode operating in the mid-wavelength infrared domain. We first focused our attention on quantum efficiency, responsivity and angular response measurements: quantum efficiency reaches $23 \%$ at $\lambda=2.1 \mu \mathrm{m}$ for $1 \mu \mathrm{m}$ thick structure. Noise under illumination measurements are also reported: noise is limited by the Schottky contribution for reverse bias voltage smaller than $1.2 \mathrm{~V}$. The specific detectivity, estimated for $2 \pi$ field-of-view and $333 \mathrm{~K}$ background temperature, was determined equal to $2.29 \times 10^{10}$ Jones for $-0,8 \mathrm{~V}$ bias voltage and $77 \mathrm{~K}$ operating temperature. [DOI: http://dx.doi.org/10.2971/jeos.2014.14022]

Keywords: Type-II superlattice, photodiode, midwave infrared, quantum efficiency, noise

\section{INTRODUCTION AND PROBLEM STATEMENT}

Type-II InAs/ GaSb superlattice (T2SL) emerges as a good candidate to complete the main infrared technologies operating in the mid-wavelength infrared (MWIR) domain, because of its large effective mass and low Auger recombination rate [1]. InAs/GaSb T2SL could potentially combine advantages of the two main MWIR technologies: HgCdTe's high operating temperature and InSb's good uniformity. In the past six years many improvements have been reported such as a $70 \%$ quantum efficiency (QE) for a T2SL focal plane array (FPA) [2](without antireflective coating, for $81 \mathrm{~K}$ operating temperature); a quantum efficiency of a T2SL photodiode was determined equal to $60 \%$ for $150 \mathrm{~K}$ operating temperature [3]; a megapixel FPA was also fabricated [4]. A lot of these results were obtained with innovating designs using nBn [5], W- [6], C-BIRD [4] or pMp [7, 8] structures. Recently an alternative pin photodiode structure using SL period with thicker InAs layer than GaSb layer ("InAs-rich" structure) was proposed to reduce the intrinsic carrier concentration (ni) [9] as compared with a symmetric design (same GaSb and InAs thickness) showing the same cut-off wavelength, and thus to reduce significantly the dark current. This promising InAs-rich structure, as confirmed by dark measurements $[9,10]$, requires a complete and rigorous radiometric characterization to assess the actual performances of this structure and to identify the limiting factors, if any.

In this paper, we describe a full characterization of the radiometric performances of this InAs-rich photodiode made of 7.5 InAs mono layers (MLs) / 3.5 GaSb MLs with a $5.48 \mu \mathrm{m}$ cutoff at $77 \mathrm{~K}$. This extensive investigation of both classical and more unusual figures of merit aims to: 1 ) assess the potential of the T2SL technology; 2) choose the axes of future technological development; 3) introduce realistic inputs in the modeling tools which predict the performance of future infrared instruments relying on T2SL technology. In Section 2 we briefly describe the growth and processing of the sample. Section 3 presents the radiometric setup. Section 4 reports the QE and responsivity measurements. Finally, under illumination noise measurements and specific detectivity values are reported in Section 5 .

\section{SAMPLE}

InAs/GaSb SL material was grown on p-type GaSb substrate by molecular beam epitaxy in a Varian Gen II reactor. Following the growth procedure presented elsewhere [10], pre- 

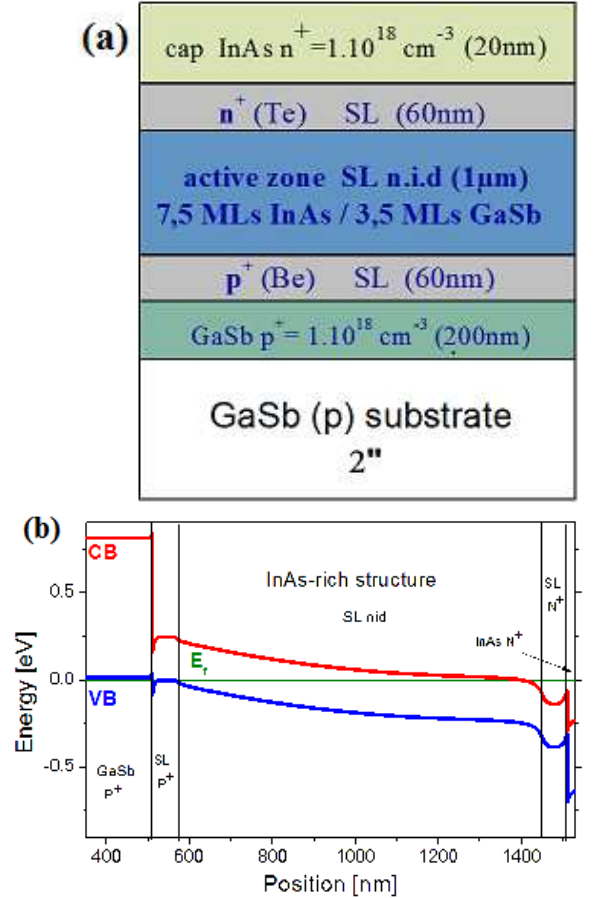

FIC. 1 (a)SL PIN structure on GaSb substrate with an active zone of $1 \mu \mathrm{m}$. (b)Schematic band structure of the studied SL.

liminary SL samples were grown and characterized to define growth parameters in order to get a perfect lattice-matching of the SL structure to the GaSb substrate. The structure (see Figure 1(a)) consists of a $200 \mathrm{~nm}$ Be-doped ( $p^{+}$-type doping $\sim 1 \times 10^{18} \mathrm{~cm}^{-3}$ ) GaSb buffer layer, several periods of $p^{+}$ doped SL, a non-intentionally doped (nid) InAs/GaSb SL active zone, several periods of $n^{+}$doped SL and a $20 \mathrm{~nm}$ Tedoped $\left(n^{+}\right.$-type doping $\sim 1 \times 10^{18} \mathrm{~cm}^{-3}$ ) InAs cap layer. The nid InAs-rich SL active region is composed of 300 periods of 7.5 InAs MLs and 3.5 GaSb MLs (7.5/3.5 SL structure), for a total thickness of $1 \mu \mathrm{m}$. Figure 1(b) presents the calculated band structure.

From epitaxial SL material, circular mesa photodiodes were obtained using standard photolithography with a mask set containing diodes with several diameters (from $20 \mu \mathrm{m}$ up to $160 \mu \mathrm{m})$. Metallizations were ensured by $\mathrm{CrAu}$ on the top of the mesa and on the back of the substrate. Mesa photodiodes were realized by chemical etching using a citric acid based solution. To complete the device processing, photoresist AZ1518 was spun onto the sample in order to protect the surface from ambient air. Then, the same mask as the one used for front-side metallization was used to open paths for wire bonding and the photoresist was heated at $200{ }^{\circ} \mathrm{C}$ for 2 hours to be polymerized. Finally, the samples were wire bounded and packaged in TO-8 sub-mounts.

Photoluminescence (PL) measurements revealed a PL peakposition at $5.48 \mu \mathrm{m}$ at $77 \mathrm{~K}$ [9]. Dark current measurements at $77 \mathrm{~K}$ are coherent with a generation-recombination limited behaviour [9]. All the following results were measured on a $160 \mu \mathrm{m}$ diameter photodiode with front-side illumination.
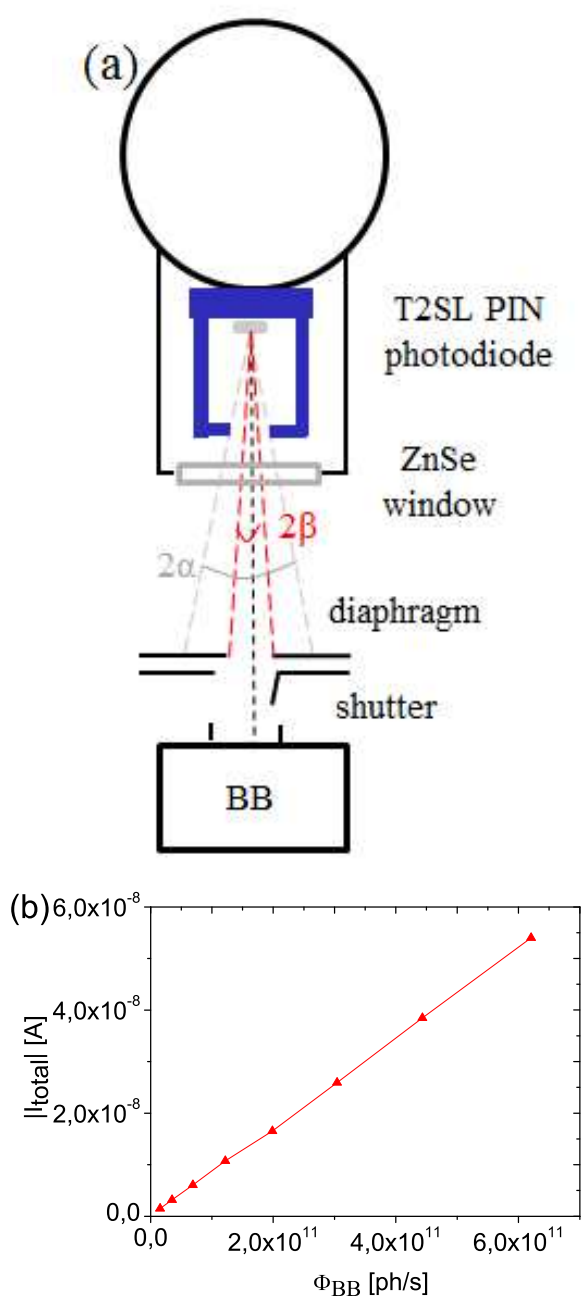

FIG. 2 (a) Experimental setup to calibrate the spectral response with a cavity blackbody. (b) Total current of InAs-rich pin photodiode versus flux for -1 $\mathrm{V}$ bias voltage at $77 \mathrm{~K}$.

\section{RADIOMETRIC CONFIGURATION}

The superlattice detector is fixed on the cold finger of a liquid nitrogen bath cryostat. A cold shield ensures a f-number $\left(=\frac{1}{2 \tan \alpha}, 2 \alpha\right.$ being the angle subtended by the pupil stop) equal to 2.9 , which is a typical value for infrared imaging applications. A ZnSe window closes the dewar, which is under vacuum. We used a $\mathrm{ZnSe}$ window with no anti-reflective coating and a constant transmission of $71 \%$ in the $0.8-20 \mu \mathrm{m}$ domain. Spectral response was measured at $77 \mathrm{~K}$ with a Keithley 428 preamplifier and a Brüker Vertex $70 \mathrm{v}$ Fourier transform infrared (FTIR) spectrometer. To deduce the absolute responsivity in $\mathrm{A} / \mathrm{W}$ or the $\mathrm{QE}$ in \%, the spectral response measurements need to be completed by measurements under rigorous controlled illumination. For this purpose, a laser source can be used [8], providing the responsivity at a given wavelength. Here we preferred to use a blackbody(BB) [11]-[13], which is more representative of the operational conditions, in terms of spectral bandwidth as well as in terms of incident power. We used a CI-systems SR-200 cavity blackbody, which operates between $T_{B B}=400{ }^{\circ} \mathrm{C}$ and $T_{B B}=1200{ }^{\circ} \mathrm{C}$. The setup is described in Figure 2(a). A diaphragm is needed because the emitting area of the BB is smaller than the cold shield aperture. This diaphragm was used to control the geometri- 
cal throughput $G$ of the detector to know precisely the flux of photons absorbed by the photodiode $\phi_{B B}$. A shutter was used to get rid of the unknown background (BG) radiations mainly due to the fluctuating temperature of the environment and to the infrared emission of the diaphragm. We measure two different currents: $I_{B G}+I_{d a r k}$, when the shutter is closed, and $I_{\text {total }}$, when the shutter is open. In Figure 2(b), we plot $I_{\text {total }}$ versus $\phi_{B B}$. A quite similar setup, using no shutter and no diaphragm but an extended BB (CI-systems SR 80, which operates between $T_{B B}=20^{\circ} \mathrm{C}$ and $T_{B B}=80^{\circ} \mathrm{C}$ ), was also developed and was already described [14]. The good agreement between the measurements with the two different setups allows us be sure to get rid of the main sources of radiometric errors: uncertainty on the BB emissivity, on the real BB temperature, existence of stray light and/or fluctuating background and uncertainty on the geometrical throughput $\mathrm{G}$ of the detector, defined as:

$$
G=\pi \times S_{d e t} \times \sin ^{2} \beta
$$

where $S_{d e t}$ is the area of the photodiode and $2 \beta$ is the angle subtended by the diaphragm.

\section{QUANTUM EFFICIENCY AND RESPONSIVITY}

The slope of the line in Figure 2(b) is $\eta_{\max } q$ as shows Eq. (2):

$$
I_{\text {total }}-\left(I_{B G}+I_{\text {dark }}\right)=\eta_{\max } \times q \times \phi_{B B}
$$

where $\eta_{\max }$ is the maximal $\mathrm{QE}$ and $\mathrm{q}$ the elementary electric charge. The absolute spectral response $R(\lambda)$ is linked with the quantum efficiency as follows:

$$
R(\lambda)=\frac{\eta(\lambda) \times q \times \lambda}{h c}
$$

The responsivity spectra are plotted in Figure 3 for different bias voltage at $77 \mathrm{~K}$. The maximal responsivity was determinated equal to $0.36 \mathrm{~A} / \mathrm{W}$ at $\lambda=2.1 \mu \mathrm{m}$ (corresponding to a maximal QE of $23 \%$ at the same wavelength) for $0 \mathrm{~V}$ bias voltage. The shape of the responsivity spectra is not surprising because this SL structure presents very wide mini-bands for the carriers, especially for the electrons, leading to density of states similar to that of a bulk material. The curves in Figure 3 exhibit two different regimes: between $\lambda=4.5 \mu \mathrm{m}$ and $\lambda=5.47 \mu \mathrm{m}$, the responsivity decreases faster than between $\lambda=2.1 \mu \mathrm{m}$ and $\lambda=4.5 \mu \mathrm{m}$. Indeed, 1) between $\lambda=2.1 \mu \mathrm{m}$ and $\lambda=4.5 \mu \mathrm{m}$, the shape of the responsivity spectra could be due to the excited interminiband transition $C_{1}-V L_{1}$, which associates the lowest electron subband $C_{1}$ to the light-hole $V L_{1}$ subband; 2) between $\lambda=4.5 \mu \mathrm{m}$ and $\lambda=5.47 \mu \mathrm{m}$, the shape of the responsivity is certainly due to the fundamental interminiband transition $C_{1}-V H_{1}$, which associates the lowest electron subband $C_{1}$ to the heavy-hole $V H_{1}$ subband. The inset in Figure 3 presents the angular response of the T2SL photodiode measured between $\theta=-70^{\circ}$ and $\theta=+70^{\circ}$ with a dedicated test bench [15]. This angular response doesn't depend on the bias voltage. Between $\theta=-70^{\circ}$ and $\theta=+70^{\circ}$, the angular response is nearly constant: it means that the photonic current delivered by an InAs/GaSb superlattice photodiode is not depending on the incident angle. This result is coherent with the fact that no coupling structure (such as diffraction gratings) needs to be used with T2SL. But this unusual

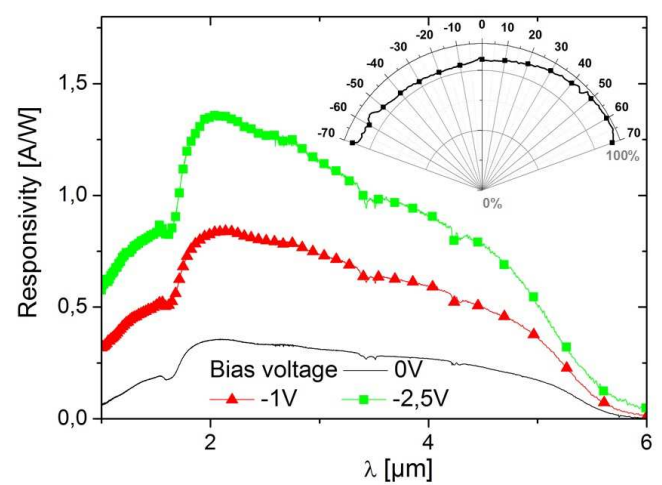

FIG. 3 Responsivity spectra of the InAs-rich SL detector for different bias voltages at 77 K. Inset: Angular response (variations of the responsivity with the angle of incidence).

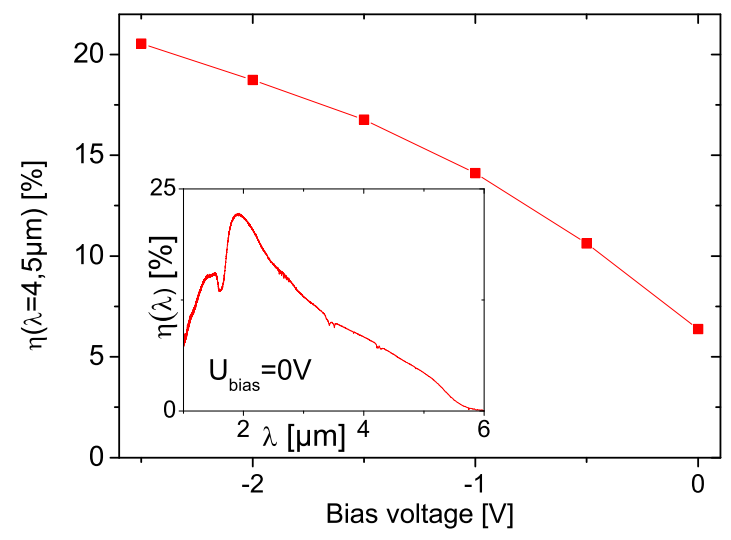

FIG. 4 Absolute quantum efficiency at $\lambda=4.5 \mu \mathrm{m}$ versus bias voltage at $77 \mathrm{~K}$. Inset: Quantum efficency spectrum for oV bias voltage at $77 \mathrm{~K}$.

figure of merit also tends to prove that the absorption of the SL active region is independent of the angle of incidence, as would be expected from a bulk material, which has never been reported up to now. As far as radiometric imaging applications are concerned, angles of incidence as high as $70^{\circ}$ can be expected for pixels located far from the optical axis. In order to retrieve a radiance calibrated image, a well known and thus predictable angular response, as obtained here, is required.

For $0 \mathrm{~V}$ bias voltage, the maximal QE is lower than the QEs in the state-of-art presented in the introduction $[2,3]$. But these performances were obtained with SL structures with thicker active zones $(2 \mu \mathrm{m})$. The absorbing zone is thicker in these devices, therefore the $\mathrm{QE}$ is higher. Moreover, these SL devices were grown with a barrier design, which exhibits higher maturity than the InAs-rich design studied in this paper.

Figure 4 presents the evolution of the quantum efficiency at $\lambda=4.5 \mu \mathrm{m}$ and $77 \mathrm{~K}$ versus bias voltage. The $4.5 \mu \mathrm{m}$ value corresponds to $\sim 80 \%$ of the cut-off wavelength and is within $3-5 \mu \mathrm{m}$ atmospheric window. The quantum efficiency spectrum for $0 \mathrm{~V}$ bias voltage is plotted in the inset in Figure 4.

The following comments can be made on Figure 4: first, QE at $4.5 \mu \mathrm{m}$ for $0 \mathrm{~V}$ bias voltage is quite low: $6.3 \%$. This value 
seems to be suprising for a SL pin photodiode. Indeed, symmetrical design with quite the same cut-off wavelength exhibited around $20 \% \mathrm{QE}$ at $4.5 \mu \mathrm{m}$ and $0 \mathrm{~V}$ [11]. Secondly, we observe an increase of the quantum efficiency with the reverse bias voltage, allowing the InAs-rich design to reach a higher QE than the symmetrical design for high reverse bias voltage. QE was also measured for other InAs-rich samples: QE always increased with the reverse bias voltage. A low $Q E$ can be due either to low absorption or to non-optimal carriers collection. We believe that the absorption of the InAs-rich design is not reduced compared to the symmetrical design, since all our symmetrical and InAs-rich samples exhibit roughly the same photoluminescence intensity. The low QE of the InAsrich SL structure at $0 \mathrm{~V}$ and the increase of the QE with reverse bias voltage, which reminds some early results on SL design with blocking barriers $[5,16]$, can thus more probably be attributed to a non-optimal collection of minority carriers. Since the n-i-d zone is n-type residual, minority carriers are holes. They are expected to exhibit lower diffusion length than electrons. Moreover the real PN junction is between the nid active zone and Be-doped ( $p+$ type doping) SL layer, far from the illuminated side of the detector, which is the InAs cap layer. In a SL structure, the absorption coefficient, which is linked to the density of states, is higher for the high energies than for the low energies. Therefore low energy photons (long wavelengths) can penetrate deeper into the structure than the high energy photons (short wavelengths). If the $\mathrm{n}-\mathrm{i}-\mathrm{d}$ zone is not completely depleted at $0 \mathrm{~V}$, a low diffusion length of the holes could explain that the carriers are not efficiently collected. Increasing the reverse bias voltage would increase the depletion width, and thus would allow more holes to be collected, leading to increased QE. This increase of the QE would be more pronounced for the short wavelengths than for the long wavelengths as observed in Figure 3 because a lot of low energy photons are absorbed near the illuminated side and far from the PN junction; these photo-excited carriers can be collected only with a large space charge region. To confirm this analysis, measurements on samples with different thicknesses are required. This study will be the subject of a forthcoming paper.

\section{NOISE UNDER ILLUMINATION}

An important figure of merit of the performances of a photodiode is the signal-to-noise ratio (SNR). The noise is often estimated as the root mean square of the Johnson noise and the Schottky noise, but some measurements in literature exhibited excess noise [17] which is critical for the SNR of the photodiode.

Up to now, noise in superlattice InAs/GaSb photodiode was often measured under dark conditions, either to find the best passivation layer [18] or to verify the potentiality of the InAs/GaSb T2SL technology [19, 20]. Some authors [17] measured the noise of a large number of pin photodiodes, to estimate the dispersion of noise performances that could be expected for a T2SL FPA. We decided to measure noise in operational conditions, in terms of incident power, namely when the photodiode is under illumination. We focused only on few good photodiodes to estimate the potential of this technology.

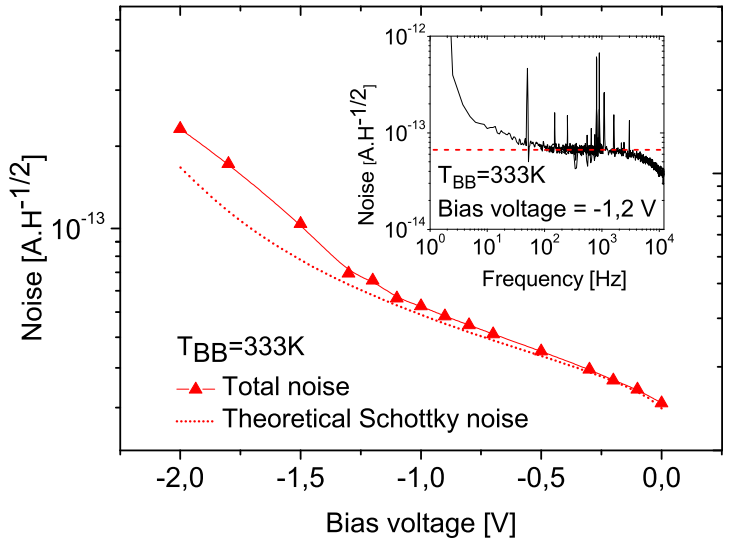

FIG. 5 Experimental intrinsic noise versus bias voltage, for a $60^{\circ} \mathrm{C}$ blackbody and $77 \mathrm{~K}$ operating temperature, and comparison with theoretical Schottky contribution (dash line). Inset: noise spectrum for $-1.2 \mathrm{~V}$ bias voltage.

A uniform extended blackbody was used to generate a wellknown, adjustable incident power on the single element. The detector noise current was converted to an output voltage by a transimpedance amplifier (TIA). We used a Femto DLPCA 200 TIA with a gain set to $10^{9}$ and a $7 \mathrm{kHz}$ bandwidth. To apply a bias voltage, we used a Fluke 5500 voltage generator. A remotely controlled ONO SOKKI spectrum analyzer, connected to the output of the TIA, measured the noise spectrum. For each measurement, 100 spectra were averaged. To avoid vibrations, which could contribute to low frequency noise, we preferred a bath cryostat to a flow-through cryostat. As well, all the measuring instruments were mechanically decoupled. Proper shielding and grounding techniques ensured that electromagnetic interference and ground-loops effects were negligible.

The total expected noise $\sigma_{i_{\text {total }}}$ (expressed in $A \cdot H z^{-1 / 2}$ ) is due to several contributions: Schottky noise $\sigma_{i_{\text {Schottky }}}$ Johnson noise $\sigma_{i_{\text {Johnson }}}, 1 / \mathrm{f}$ noise $\sigma_{i_{1 / f}}$ and setup noise $\sigma_{i_{\text {setup }}} . \sigma_{i_{\text {total }}}$ is given by:

$$
\sigma_{i_{\text {total }}}^{2}=\sigma_{i_{\text {Schottky }}}^{2}+\sigma_{i_{\text {Johnson }}}^{2}+\sigma_{i_{1 / f}}^{2}+\sigma_{i_{\text {setup }}}^{2}
$$

The expected spectrum is divided into three parts: at low frequencies $1 / \mathrm{f}$ noise is the most important; then comes a white noise plateau; at higher frequencies the signal (total noise) drops because of the cutoff of the amplifier.

Figure 5 presents the experimental intrinsic noise versus the bias voltage for $333 \mathrm{~K} \mathrm{BB}$ temperature. The inset in Figure 5 is an experimental noise spectrum for the same BB temperature at $-1.2 \mathrm{~V}$ bias voltage. Up to $-1,3 \mathrm{~V}$ bias voltage, the intrinsic noise is quite comparable to the Schottky contribution. The good agreement between experiment and theory confirms the excellent crystallographic quality of the InAs-rich InAs/GaS pin SL structure. An excess noise appears for $U_{\text {bias }} \leq-1.2 \mathrm{~V}$, but our measurements clearly show that, for typical bias voltage used in focal plane arrays (a few tens of millivolts), no extra noise is observed.

With the previous measurements, we can estimate the real 


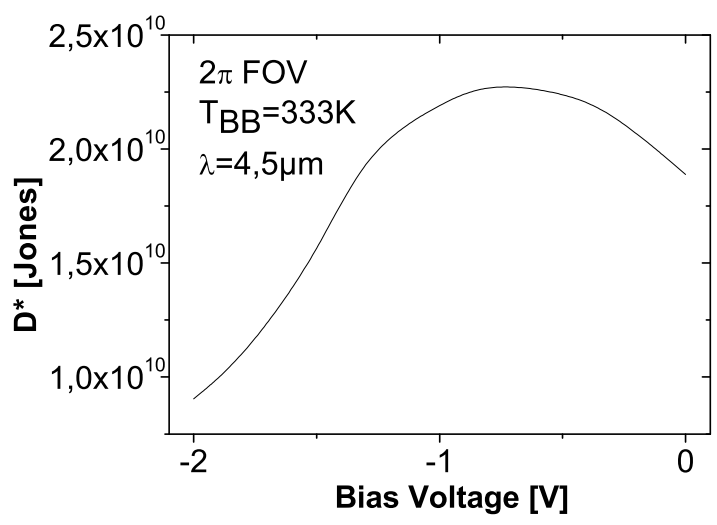

FIG. 6 Specific detectivity for $77 \mathrm{~K}$ operating temperature versus bias voltage estimated for $2 \pi$ field-of-view (FOV) and $333 \mathrm{~K}$ background temperature.

specific detectivity $D^{\star}$ :

$$
\begin{aligned}
D^{\star} & =R(\lambda) \times \frac{\sqrt{S_{\text {det }}}}{\sigma_{i_{\text {total }}}} \\
& =\frac{\eta(\lambda) \times q \times \lambda}{h c} \times \frac{\sqrt{S_{\text {det }}}}{\sqrt{\sigma_{i_{\text {Schottky }}^{2}}^{2}+\sigma_{i_{\text {Johnson }}}^{2}+\sigma_{i_{1 / f}}^{2}}}
\end{aligned}
$$

where the specific detectivity is expressed in Jones, $S_{\text {det }}$ is the area of the pixel (in $\mathrm{cm}^{2}$ ), $R(\lambda)$ is the responsivity measured in Section 4 (see Figure 3 ), and $\sigma_{i_{\text {total }}}$ is the noise of the detector (in $A \cdot \sqrt{\mathrm{Hz}}$ ) measured in Section 5 (see Figure 5). Specific detectivity is often determined in the literature with noise values measured under dark conditions or with theoretical values [5]. Figure 6 presents the specific detectivity estimated for $2 \pi$ field-of-view (FOV) at $\lambda=4.5 \mu \mathrm{m}$ under illumination: the blackbody temperature is equal to $60{ }^{\circ} \mathrm{C}$. The maximum value of the specific detectivity $\left(2.29 \times 10^{10}\right.$ Jones $)$ is obtained for $-0,8 \mathrm{~V}$ bias voltage, which is quite unusual but remains compatible with the bias voltage in a read-out integrated circuit; this maximum value is not reached at $0 \mathrm{~V}$ bias voltage as we would expect, because the QE increases faster than the total noise with the reverse bias voltage until $U_{\text {bias }}=-0.8 \mathrm{~V}$. For $U_{\text {bias }} \leq-0.8 \mathrm{~V}$, the specific detectivity decreases with the reverse bias voltage because the total noise increases faster than the QE. At $-0.8 \mathrm{~V}$, the $D^{\star}$ value is slightly lower than T2SL state-of-art values [21]. The background-limited $D^{\star}$ (with $T_{B B}=300 \mathrm{~K}$ ) at $4 \mu \mathrm{m}$ for an ideal photovoltaic detector is one order of magnitude higher [22]. This difference is probably due to the low quantum efficiency for low reverse bias voltage. Moreover Figure 6 presents a $D^{\star}$ under illumination: the total current and therefore the Schottky noise are higher under illumination than in dark conditions; therefore the $D^{\star}$ measured with $333 \mathrm{~K}$ background temperature is lower than the $D^{\star}$ measured with $300 \mathrm{~K}$ background temperature or estimated in dark conditions.

\section{CONCLUSION}

We reported the full electro-optical characterization of $1 \mu \mathrm{m}$ thick InAs-rich InAs/GaSb type-II superlattice PIN photodiode. We determined the quantum efficiency for $77 \mathrm{~K}$ oper- ating temperature, which reaches $23 \%$ at $\lambda=2.1 \mu \mathrm{m}$ and $6.3 \%$ at $\lambda=4.5 \mu \mathrm{m}$ for $0 \mathrm{~V}$ bias voltage. This rather low value is a signature of an imperfect collection of minority carriers. This could be due to a non optimised design of the structure. Our measurements also revealed a rather flat angular response, as would be expected from a bulk material. The noise under illumination is limited by the Schottky contribution for reverse bias voltage smaller than $1.2 \mathrm{~V}$. Our measurements confirm the good crystallographic and technological quality of the InAs/GaSb superlattice pin photodiodes, since no excess noise was observed in the operating conditions of MWIR FPA.The specific detectivity is slightly lower than the state-of-art, which is consistent with the rather low QE. Up to now, the realized measurements stand T2SL in good stead for high performance applications such as radiometric imagers but they have to be completed by other measurements like, for example, non uniformity and stability over time.

\section{References}

[1] E. Youngdale, J. Meyer, C. Hoffman, F. Bartoli, C. Grein, P. Young, H. Ehrenreich, et al., "Auger lifetime enhancement in InAs-Ga1xInxSb superlattices," Appl. Phys. Lett. 64, 3160-3162 (1994).

[2] E. K.-W. Huang, S. Abdollahi Pour, M.-A. Hoang, A. Haddadi, M. Razeghi, and M. Z. Tidrow, "Low irradiance background limited type-II superlattice MWIR M-barrier imager," 0pt. Lett. 37, 2025-2027 (2012).

[3] M. Razeghi, S. Abdollahi Pour, E. K. Huang, G. Chen, A. Haddadi, and B. M. Nguyen, "Type-II InAs/GaSb photodiodes and focal plane arrays aimed at high operating temperatures," Opto-Electron. Rev. 19, 261-269 (2011).

[4] S. D. Gunapala, D. Z. Ting, C. J. Hill, J. Nguyen, A. Soibel, S. B. Rafol, S. A. Keo, et al., "Demonstration of $1 \mathrm{KX} 1 \mathrm{~K}$ long-wave and midwave superlattice infrared focal plane arrays," Proc. SPIE 7808, 780802-780802 (2010).

[5] J. B. Rodriguez, E. Plis, G. Bishop, Y. D. Sharma, H. Kim, L. Dawson, and S. Krishna, "nBn structure based on InAs/ GaSb type-II strained layer superlattices," Appl. Phys. Lett. 91, 043514 (2007).

[6] E. H. Aifer, I. Vurgaftman, C. L. Canedy, J. H. Warner, E. M. Jackson, J. G. Tischler, and J. R. Meyer, "Recent progress in W-structured type-II superlattice photodiodes," Proc. SPIE 6479, 64790Y-64790Y (2007).

[7] S. Abdollahi Pour, E. K.-w. Huang, G. Chen, A. Haddadi, B.-M. Nguyen, and M. Razeghi, "High operating temperature midwave infrared photodiodes and focal plane arrays based on type-II InAs/GaSb superlattices," Appl. Phys. Lett. 98, 143501 (2011).

[8] B.-M. Nguyen, G. Chen, A. M. Hoang, S. A. Pour, S. Bogdanov, and M. Razeghi, "Effect of contact doping in superlattice-based minority carrier unipolar detectors," Appl. Phys. Lett. 99, 033501 (2011).

[9] J.-B. Rodriguez, C. Cervera, and P. Christol, "A type-II superlattice period with a modified InAs to Gasb thickness ratio for midwavelength infrared photodiode performance improvement," Appl. Phys. Lett. 97, 251113 (2010).

[10] R. Taalat, J.-B. Rodriguez, M. Delmas, and P. Christol, "Influence of the period thickness and composition on the electro-optical properties of type-II InAs/GaSb midwave infrared superlattice photodetectors," J. Phys. D Appl. Phys. 47, 015101 (2014). 
[11] R. Rehm, M. Walther, J. Schmitz, J. Fleißner, F. Fuchs, J. Ziegler, and W. Cabanski, "InAs/GaSb superlattice focal plane arrays for high-resolution thermal imaging," Opto-Electron. Rev. 14, 19-24 (2006).

[12] E. Plis, J.-B. Rodriguez, G. Balakrishnan, Y. D. Sharma, H. S. Kim, T. Rotter, and S. Krishna, "Mid-infrared InAs/GaSb strained layer superlattice detectors with $\mathrm{nBn}$ design grown on a CaAs substrate," Semicond. Sci. Tech. 25, 085010 (2010).

[13] J. W. Little, S. P. Svensson, W. A. Beck, A. C. Goldberg, S. W. Kennerly, T. Hongsmatip, M. Winn, and P. Uppal, "Thin active region, type II superlattice photodiode arrays: Single-pixel and focal plane array characterization," J. Appl. Phys. 101, 044514 (2007).

[14] I. Ribet-Mohamed, M. Tauvy, R. Taalat, C. Cervera, J.-B. Rodriguez, and P. Christol, "Comparison of the electro-optical performances of symmetrical and asymmetrical MWIR InAs/CaSb superlattice pin photodiodes," Proc. SPIE 8268, 826833-826833 (2012).

[15] I. Ribet-Mohamed, J. Le Rouzo, S. Rommeluere, M. Tauvy, and N. Guérineau, "Advanced characterization of the radiometric performances of quantum well infrared photodetectors," Infrared Phys. Techn. 47, 119-131 (2005).

[16] I. Vurgaftman, E. H. Aifer, C. L. Canedy, J. G. Tischler, J. R. Meyer, J. H. Warner, E. M. Jackson, et al., "Graded band gap for darkcurrent suppression in long-wave infrared W-structured type-II superlattice photodiodes," Appl. Phys. Lett. 89, 121114 (2006).
[17] A. Wörl, P. Kleinow, R. Rehm, J. Schmitz, and M. Walther, "Noise characteristics of InAs/GaSb superlattice infrared photodiodes," Phys. Status Solidi C 10, 744-747 (2013).

[18] T. Tansel, K. Kutluer, 0. Salihoglu, A. Aydinli, B. Aslan, B. Arikan, M. C. Kilinc, et al., "Effect of the passivation layer on the noise characteristics of mid-wave-infrared InAs/GaSb superlattice photodiodes," IEEE Photonic. Tech. L. 24, 790-792 (2012).

[19] K. Jaworowicz, I. Ribet-Mohamed, C. Cervera, J.-B. Rodriguez, and P. Christol, "Noise characterization of midwave infrared InAs/GaSb superlattice pin photodiode," IEEE Photonic. Tech. L. 23, 242-244 (2011).

[20] C. Cervera, I. Ribet-Mohamed, R. Taalat, J. Perez, P. Christol, and J. Rodriguez, "Dark current and noise measurements of an InAs/GaSb superlattice photodiode operating in the midwave infrared domain," J. Electron. Mater. 41, 2714-2718 (2012).

[21] A. Rogalski, J. Antoszewski, and L. Faraone, "Third-generation infrared photodetector arrays," J. Appl. Phys. 105, 091101 (2009).

[22] A. Rogalski, "Infrared detectors: status and trends,"Prog. Quant. Electron. 27, 59-210 (2003). 